\title{
Familial calcium pyrophosphate deposition
}

INSERM

\section{Source}

INSERM. (1999). Orphanet: an online rare disease and orphan drug data base. Familial calcium pyrophosphate deposition. ORPHA:1416

Familial calcium pyrophosphate deposition (CPPD) is a chronic inherited arthropathy characterized by chondrocalcinosis (CC; i.e. cartilage calcification), often associated with recurrent acute calcium pyrophosphate (CPP) crystal arthritis and polyarticular osteoarthritis (OA). 